\title{
Nonlinear gravito-electrostatic waves in self-gravitating complex plasma in presence of ion-drag effects
}

\author{
A. Haloi ${ }^{1}$ P.K. Karmakar ${ }^{1}$
}

Received: 7 April 2015 / Accepted: 9 July 2015 / Published online: 25 July 2015

(c) The Author(s) 2015. This article is published with open access at Springerlink.com

\begin{abstract}
We present theoretical model analysis to study fully nonlinear behavior of gravito-electrostatic fluctuations in unmagnetized self-gravitating collisional dust cloud in presence of the ion-drag forces methodologically on the Jeans scales of space and time. The ion-drag effect as a result of streaming plasma ions arises here due to the ion orbital motion (scattering effect by dust) and the ion momentum transfer (capturing effect by dust) processes in opposite phase with the electrostatic force field. All the realistic astrophysical processes, such as electron impact-ionization of the neutral atoms, volume recombination, attachment of the electrons and ions to the dust grains and the collective plasma particle collisions are jointly considered. The Sagdeev pseudo-potential formulation is methodologically carried out in modified form to derive a new pair of gravitoelectrostatically coupled energy integral equations. A numerical analysis is made to see the fluctuation features in judicious plasma parameter window. It is shown that the fluctuation dynamics evolves as self-gravitational rarefactive solitary structures and electrostatic compressive shocklike spectral patterns. The new features brought about by the considered ion-drag effects are discussed in the light of the existing theoretical, experimental and satellite-based predictions. The relevance of our results to understand the dynamics of self-gravitational collapse leading to galactic structure formation in interstellar space is briefly summarized.
\end{abstract}

Keywords Dust cloud · Ion-drag force $\cdot$ Sagdeev potential $\cdot$ Nonlinear structures

P.K. Karmakar

pkk@tezu.ernet.in

1 Department of Physics, Tezpur University, Napaam 784028, Tezpur, Assam, India

\section{Introduction}

The study of nonlinear gravito-electrostatic waves, oscillations and fluctuations in self-gravitating dusty plasmas in the form of dust molecular clouds (DMCs) has been an importantly emerging area of great interest for years. The growing interest is mainly due to the roles played by such fluctuations in the dynamics of self-gravitational collapse leading to the formation of bounded equilibrium structures in diverse astrophysical situations. Such structures, to name a few, include stars, stellar rings, planets, planetesimals, planetary rings, cometary tails, clusters, etc. (Mace and Hellberg 1993; Mamun and Shukla 2002; Shukla and Mamun 2003; Paul et al. 2006; Karmakar et al. 2012).

It is seen that many authors have studied the evolutionary dynamics of various waves in different dusty plasma configurations with wide-range existence from laboratory to space in past. They have found various structural waves which can either modify the original waves and instabilities or introduce new eigenmodes like solitons, shocks, double layers, vortices, etc. (Rao et al. 1990; Shukla and Silin 1992; Mace and Hellberg 1993; Mamun 1999; Shukla and Mamun 2002, 2003). The dynamics of these waves have been investigated theoretically (Rao et al. 1990; Shukla and Silin 1992; Mace and Hellberg 1993; Shukla and Mamun 2003) in diverse plasma situations. Rao et al. have theoretically predicted the existence of the low-frequency dust-acoustic wave (DAW) in an unmagnetized dusty plasma by using the reductive perturbation technique (Rao et al. 1990). They have showed that these waves propagate linearly as normal sound modes and nonlinearly as soliton-like eigenmodes, which arise due to the balance between nonlinear wave steepening and linear dispersion (Rao et al. 1990). On the other hand, Shukla and Silin theoretically confirmed the existence of the low-frequency DAW (Shukla and Silin 1992) 
in weakly unmagnetized dusty plasma. Mamun has investigated both weakly and strongly nonlinear waves in unmagnetized dusty plasma by using both the reductive perturbation and the Sagdeev pseudo-potential approaches (Mamun 1999). He has considered a three-component fluid model consisting of the inertial charged dust grains and the Boltzmann distributed electrons and ions. It has been found that both weakly and strongly nonlinear waves admit solitary structure only with negative potential. Shukla et al. have also theoretically investigated the dust-acoustic shock in strongly coupled unmagnetized dusty plasma (Shukla and Mamun 2001) and so forth.

Several authors have investigated the dynamics of these nonlinear eigenmodes considering self-gravity in dusty plasma (Khare and Shukla 1994; Pandey and Dwivedi 1996; Burman and Chowdhury 2002; Pandey et al. 2003; Misra et al. 2004, 2005; Misra and Chowdhury 2006; Karmakar and Borah 2012). The self-gravitational effect arises due to the micron-sized massive dust grains, which balance the electrostatic repulsive effect and induce coupled gravito-electrostatic fluctuations. As a result, different nonlinear eigenmodes, i.e., solitons, shocks, double layers, vortices, etc. evolve, which help us in explaining the basic physics behind the star formation, the particleacceleration, material-transportation and self-gravitational collapse mechanism in astrophysical situations including interplanetary dust, galaxies, planetary rings, etc. (Blandford and Ostriker 1978; Khare and Shukla 1994; Burman and Chowdhury 2002; Pandey et al. 2002, 2003; Bergin et al. 2004; Misra et al. 2005; Ivlev et al. 2010; Carley et al. 2013).

The excitations of these nonlinear eigenmodes have also been seen experimentally (Luo et al. 1999; Reighard et al. 2006; Sheridan et al. 2008; Bandyopadhyay et al. 2008; Merlino et al. 2012), and by satellite (like Viking, FAST, Freja, Polar, Cluster spacecraft, etc.) observations (Gosling et al. 1968; Ergun et al. 1998a; Ergun et al. 1998b; Franz et al. 1998; Lee et al. 2009; Pickett et al. 2003; Carley et al. 2013) in presence of the dust grains. Lue et al. have studied experimentally the formation of the nonlinear shock structures in dusty plasma (Lue et al. 1999), whereas, Reighard et al. have also observed like structures in different experimental conditions (Reighard et al. 2006). Bandyopadhyay et al. have experimentally studied the excitation and propagation of the solitary waves in Argon plasma with Kaolin dust grains (Bandyopadhyay et al. 2008). They have found the nonlinear solitary waves excited by pulse modulating voltage with electrostatic negative potential.

The instabilities or fluctuations giving rise to the nonlinear structures may also arise in dusty plasma due to the ion-drag force, ionization, dust-charge fluctuation and so on (D'Angelo 1998; Misra et al. 2004). Recently, a number of authors have studied the instabilities of the DAW in presence of the ion-drag force, ionization, dust charge fluctuation, etc. in dusty plasma (D'Angelo 1998; Ivlev et al. 1999, 2004). The ion-drag force arises due to the ion orbital motion around the grains, as well as the momentum transfer from the ions which are captured by the grains. It plays a crucial role in understanding the location and configuration of the grains, interaction between the grains, instabilities and nonlinear fluctuations in space and laboratory dusty plasmas. D'Angelo has theoretically investigated damped DAW fluctuations in a uniform, unmagnetized, four-component plasma consisting of positive ions, electrons, negatively charged dust grains and neutral atoms in presence of the ion-drag force (D'Angelo 1998). Accordingly, later Ivlev et al. have shown that the acoustic modes can be unstable in the presence of the ion-drag forces and ionization effects (Ivlev et al. 2004).

The main motive behind this work is to study the fully nonlinear behavior of the gravito-electrostatic eigenmodes (in 1-D geometry) in the DMC in presence of the iondrag force effects. All physical processes, such as ionization, recombination, dust-charge relaxation and whole collisional dissipative agencies are taken into account. Applying the Sagdeev pseudo-potential approach (Sagdeev 1966; Mace and Hellberg 1993; Mamun 1999; Shukla and Mamun 2003), it is methodologically shown that the electrostatic eigenmodes evolve as compressive shock-like structures and the self-gravitational eigenmodes germinate as rarefactive soliton-like patterns, governed by a new pair of gravito-electrostatically coupled energy integral equations. The outline of the paper is organized as follows. Apart from introduction already presented in Sect. 1, Sects. 2 and 3 describe our physical and the mathematical models, respectively. Section 4 includes the properties of the arbitrary amplitude gravito-electrostatic fluctuation eigenmodes. Section 5 presents the numerical results and discussions. Finally, Sect. 6 depicts the main conclusions derived from our studies together with futuristic expansion highlights.

\section{Physical model}

We consider a one-dimensional (1-D), unmagnetized, selfgravitating and collisional DMC consisting of the warm electrons, positive ions and negatively charged identical dust grains (partially ionized) with presumed global quasineutrality condition. The plasma model includes the iondrag forces arising due to electro-mechanical ion-grain interaction on the Jeans scales of space and time. These dragforces act in anti-phase with the bipolar electrostatic forces arising due to ambipolar diffusion mechanism. More simply speaking, the ion-drag forces arise due to the orbital motion of the ions around the grains and the momentum transfer from the ions, which are collected by the grains. The charge 
of the grains in interstellar media, in reality, varies with space and time due to the electron-ion sticking mechanism, secondary electron emission, photo-emission, thermionic emission and so forth (Shukla and Mamun 2002). However, for convenience in mathematical simplifications, we consider the dust charge as a static parameter. This is based on the justified approximation that the dust charging time scale $\left(10^{-8} \mathrm{~s}\right)$ is much smaller than the characteristic evolutionary time scale $\left(10^{-3} \mathrm{~s}\right)$ of the considered fluctuation dynamics (Pandey and Dwivedi 1996). Besides, the background neutrals are assumed to be immobile on the time scale of the wave dynamics of our interest.

On the Jeans scales of space and time, the electrons and ions are assumed to be thermalized with the corresponding temperature-scaling $T_{e} \approx T_{i}=T_{p} \gg T_{d}$ (in $\mathrm{eV}$ ), dictated by the asymmetric mass-scaling $m_{e}<m_{i} \ll m_{d}$, the conventional symbols used are discussed later. All the physical processes of astrophysical relevance, such as, ion-drag forces, ionization-recombination processes and collisional dissipation effects are also included. The dissipative or damping effects here come from the various collisions between the electrons-neutrals, ions-neutrals, electrons-ions, etc.

\section{Mathematical analysis}

The cloud dynamics under consideration is described by a modified model set of conservative fluid equations in gravito-electrostatic equilibrium condition. As already discussed, it considers all the realistic effects like ion-drag forces, ionization, recombination, attachment of electrons and ions to the dust grains, etc. Thus, the cloud dynamical evolution is completely dictated by the continuity equations, momentum equations and the closing electro-gravitational Poisson equations in a planar geometry (Misra et al. 2004). The basic governing equations describing our physical model in unnormalized form are enlisted respectively as follows:

$$
\begin{aligned}
& \frac{\partial n_{e}}{\partial t}+\frac{\partial\left(n_{e} u_{e}\right)}{\partial x}=S_{e}, \\
& \frac{\partial u_{e}}{\partial t}+v_{e}^{\mathrm{eff}} u_{e}+\frac{T_{e}}{m_{e} n_{e}} \frac{\partial n_{e}}{\partial x}=\frac{e}{m_{e}} \frac{\partial \phi}{\partial x}, \\
& \frac{\partial n_{i}}{\partial t}+\frac{\partial\left(n_{i} u_{i}\right)}{\partial x}=S_{i}, \\
& \frac{\partial u_{i}}{\partial t}+v_{i}^{\mathrm{eff}} u_{i}+\left(\frac{T_{i}}{m_{i} n_{i}}\right) \frac{\partial n_{i}}{\partial x}=-\frac{Z_{i} e}{m_{i}} \frac{\partial \phi}{\partial x}, \\
& \frac{\partial n_{d}}{\partial t}+\frac{\partial\left(n_{d} u_{d}\right)}{\partial x}=0, \\
& \frac{\partial u_{d}}{\partial t}+u_{d} \frac{\partial u_{d}}{\partial x}+v_{\mathrm{dn}} u_{d}+\left(\frac{T_{d}}{m_{d} n_{d}}\right) \frac{\partial n_{d}}{\partial x} \\
& \quad=-\left(\frac{q_{d}}{m_{d}}\right) \frac{\partial \phi}{\partial x}+\frac{F_{\mathrm{di}}}{m_{d}}-\frac{\partial \psi}{\partial x},
\end{aligned}
$$

$$
\begin{aligned}
& \frac{\partial^{2} \psi}{\partial x^{2}}=4 \pi G m_{d} n_{d}, \quad \text { and } \\
& \frac{\partial^{2} \phi}{\partial x^{2}}=-4 \pi e\left\{\left(Z_{i} n_{i}\right)-\left(n_{e}\right)+\left(\frac{q_{d}}{e}\right) n_{d}\right\} .
\end{aligned}
$$

where, $n_{j}, u_{j}, m_{j}$ and $T_{j}$ (in eV) are the population density, velocity, mass and temperature of the plasma species, where $j=e$ for the electrons, $i$ for the ions and $d$ for the dust grains, such that $T_{e} \approx T_{i}=T_{p} \gg T_{d}$; respectively. The notations $\phi$ and $\psi$ are respectively the electrostatic and self-gravitational potentials. The source terms in Eqs. (1) and (3) are defined as $S_{e}=-v_{\mathrm{ed}} n_{e}+v_{i} n_{e}-V_{\mathrm{eff}} n_{e}^{2}$ and $S_{i}=-v_{\mathrm{id}} n_{i}+v_{i} n_{e}-V_{\mathrm{eff}} n_{e}^{2}$, where, $v_{\mathrm{ed}}$ and $v_{\mathrm{id}}$ represent the attachment frequencies of the electrons and ions to the grains, respectively. Also, $v_{i}$ is the rate for electron impact-ionization of the neutral atoms and $V_{\text {eff }}$ is the difference between the volume recombination rate and the stepwise ionization rate. For more details, interested readers are suggested to go through the book by Bittencourt (Bittencourt 2004). Then, the symbol $v_{\mathrm{dn}}$ is for the effective collisional frequency between the grains and neutrals. Besides, $v_{e}^{\text {eff }}$ is the effective collision frequency in momentum exchange processes between electron-ion, electron-neutral, ion-neutral, elastic and inelastic electron-ion impacts with the grains. The notation $F_{\text {id }}$ represents the ion-drag force, $q_{d}=-Z_{d} e$ is the dust charge with $e$ as the electronic charge, and all the rest, having their usual significances (Shukla and Mamun 2002; Misra et al. 2004).

As already mentioned, the principal goal of this paper is centered on investigation of fully nonlinear gravitoelectrostatic waves in presence of the complex ion-drag force influences in the plasma parameter regimes previously remaining unexplored. The ion-drag force by the streaming plasma ions is well-known to consist of two distinct components-collection force (capturing effect by the grains, but not scattering) and orbital force (scattering effect by the grains, but no capturing). Thus, the explicit unnormalized form of the resultant ion-drag force with all the usual notations (Shukla and Mamun 2002; Misra et al. 2004) can analytically be derived and summarily written as,

$F_{\mathrm{di}}=4 \pi n_{i} m_{i} V_{\mathrm{it}} v_{i} b_{\pi / 2}^{2} \wedge\left(V_{\mathrm{it}}\right)$,

where, $V_{\mathrm{it}}=\left(v_{i}^{2}+8 V_{\mathrm{Ti}}^{2} / \pi\right)^{1 / 2}$ is the resultant ion-flow speed, which is the sum of the directed drift speed, $v_{i}$ and the random thermal speed, $V_{\mathrm{Ti}}$. Also, $b_{\pi / 2}=Z_{d} e^{2} / m_{i} V_{\mathrm{it}}^{2}$ is the orbital impact parameter for the $\theta=\pi / 2$ deflection relative to the initial ion-flow. Next, $\wedge\left(V_{\mathrm{it}}\right)=\ln \left[\left(\lambda_{\mathrm{De}}^{2}+b_{\pi / 2}^{2}\right) /\left(b_{c}^{2}+\right.\right.$ $\left.\left.b_{\pi / 2}^{2}\right)\right]^{1 / 2}$ is the Coulomb logarithm, analytically obtained by integrating spatially from the collection impact parameter, $b_{c}=r_{d}\left(1+2 Z_{d} e^{2} / r_{d} m_{i} V_{\mathrm{it}}^{2}\right)$ to the electron plasma Debye length, $\lambda_{\text {De }}$. The other symbols, like $r_{d}$ and $Z_{d}$, stand for the dust grain radius and grain surface charge number under identical spherical grain approximation. 
The normalized closed set of basic governing Eqs. (1)(8), after a standard astrophysical normalization procedure being discussed later, is set out as below:

$$
\begin{aligned}
& \frac{\partial N_{e}}{\partial \tau}+\frac{\partial M_{e} N_{e}}{\partial \xi}=-f_{\mathrm{ed}} N_{e}+f_{i} N_{e}-V_{\mathrm{eff} 0} n_{e} N_{e}^{2}, \\
& \frac{\partial M_{e}}{\partial \tau}+f_{e}^{\mathrm{eff}} M_{e}+\alpha \frac{1}{N_{e}} \frac{\partial N_{e}}{\partial \xi}=\alpha \frac{\partial \Phi}{\partial \xi}, \\
& \frac{\partial N_{i}}{\partial \tau}+\frac{\partial M_{i} N_{i}}{\partial \xi}=-f_{\mathrm{id}} N_{i}+f_{i} N_{e}\left(\frac{n_{e 0}}{n_{i 0}}\right) \\
& -V_{\mathrm{eff} 0}\left(\frac{n_{e 0}^{2}}{n_{i 0}}\right) N_{e}^{2}, \\
& \frac{\partial M_{i}}{\partial \tau}+f_{i}^{\mathrm{eff}} M_{i}+\beta\left(\frac{T_{i}}{T_{e}}\right) \frac{1}{N_{i}} \frac{\partial N_{i}}{\partial \xi}=-Z_{i} \beta \frac{\partial \Phi}{\partial \xi}, \\
& \frac{\partial N_{d}}{\partial \tau}+\frac{\partial\left(N_{d} M_{d}\right)}{\partial \xi}=0, \\
& \frac{\partial M_{d}}{\partial \tau}+M_{d} \frac{\partial M_{d}}{\partial \xi}+f_{\mathrm{dn}} M_{d}+\left(\frac{T_{d}}{T_{e}}\right) \frac{1}{N_{d}} \frac{\partial N_{d}}{\partial \xi} \\
& =-\left(\frac{q_{d}}{e}\right) \frac{\partial \Phi}{\partial \xi}+F_{N}^{\mathrm{di}}-\frac{\partial \Psi}{\partial \xi}, \\
& \frac{\partial^{2} \Psi}{\partial \xi^{2}}=\frac{4 \pi G m_{d} n_{d 0}}{\omega_{J}^{2}} N_{d},
\end{aligned}
$$

and

$\frac{\partial^{2} \Phi}{\partial \xi^{2}}=-\frac{4 \pi e^{2}}{m_{d} \omega_{J}^{2}}\left\{\left(Z_{i} n_{i 0} N_{i}\right)-\left(n_{e 0} N_{e}\right)-\left(\frac{q_{d}}{e}\right) n_{d 0} N_{d}\right\}$.

Here, $\alpha=m_{d} / m_{e}$ and $\beta=m_{d} / m_{i}$ represent the asymptotic mass ratios of the grains to the electrons and ions, respectively. The independent variables like position $(\xi)$ and time $(\tau)$ are normalized by the Jeans length $\left(\lambda_{J}\right)$ and Jeans time $\left(\omega_{J}^{-1}\right)$, respectively. The parameters $N_{e}, N_{i}$ and $N_{d}$ are the normalized population densities of the electrons, ions and grains, respectively; normalized by their respective equilibrium values (viz., $n_{i 0}, n_{e 0}$ and $n_{d 0}$ ). Also, their respective fluid velocities $M_{e}, M_{i}$ and $M_{d}$ are normalized by the dust-acoustic phase speed $c_{\mathrm{ss}}$. Moreover, all the collisional frequencies $f_{\text {ed }}, f_{\text {id }}, f_{\text {dn }}$ and $f_{e}^{\text {eff }}$ are normalized by the Jeans frequency $\omega_{J}=\left(4 \pi \rho_{0} G\right)^{1 / 2}$, where $\rho_{0}=m_{d} n_{d 0}$ is the equilibrium matter density of the cloud. The electrostatic potential $\Phi$ is normalized by the cloud thermal potential $T_{p} / e$ and the self-gravitational potential $\Psi$ is normalized by $c_{\mathrm{ss}}^{2}$. Furthermore, $V_{\text {eff }}$ is the recombination coefficient normalized by the Jeans frequency $\omega_{J}$ and the ion drag force $\left(F_{N}^{\mathrm{di}}\right)$ is normalized by the thermal force $T_{p} / \lambda_{J}$. The adopted normalization scheme, for convenience of quick reference, is mathematically summarized as follows:

$$
\begin{aligned}
& \xi=x / \lambda_{J}, \quad \tau=t / \omega_{J}^{-1}, \quad N_{e}=n_{e} / n_{e 0}, \\
& N_{i}=n_{i} / n_{i 0}, \quad N_{d}=n_{d} / n_{d 0}, \quad M_{e}=u_{e} / c_{\mathrm{ss}},
\end{aligned}
$$

$$
\begin{aligned}
& M_{i}=u_{i} / c_{\mathrm{ss}}, \quad M_{d}=u_{d} / c_{\mathrm{ss}}, \quad c_{\mathrm{ss}}=\sqrt{T_{p} / m_{d}}, \\
& f_{\mathrm{ed}}=v_{\mathrm{ed}} / \omega_{J}, \quad f_{\mathrm{id}}=v_{\mathrm{id}} / \omega_{J}, \quad f_{e}^{\mathrm{eff}}=v_{e}^{\text {eff }} / \omega_{J}, \\
& f_{i}=v_{i} / \omega_{J}, \quad \Phi=e \phi / T_{p}, \quad \Psi=\psi / c_{\mathrm{ss}}, \\
& V_{\mathrm{eff} 0}=V_{\mathrm{eff}} / \omega_{J}^{-1} \quad \text { and } \quad F_{N}^{\mathrm{di}}=F^{\mathrm{di}} \lambda_{J} / T_{p} .
\end{aligned}
$$

Let us now derive a simplified normalized form of the complicated ion-drag force from Eq. (9) explicitly as a function of the relevant plasma parameters and calculate its typical numerical value in normal astrophysical cloud conditions without using any questionable rough approximation (Yaroshenko et al. 2007; Huba 2013) as follows,

$F_{N}^{\mathrm{di}}\left(N_{i}, M_{i}, Z_{d}\right)=10^{-6}\left[\frac{N_{i} Z_{d}}{M_{i}^{2}}\right]$.

In the derivation of the above equation, different parameter values used are $n_{i 0}=2 \times 10^{5} \mathrm{~m}^{-3}, n_{d 0}=1.39 \times 10^{1} \mathrm{~m}^{-3}$, $m_{i}=1.67 \times 10^{-27} \mathrm{~kg}, \wedge\left(V_{\mathrm{it}}\right) \sim 10$ and $T_{p}=10 \mathrm{eV}$.

\section{The Sagdeev pseudo-potential approach}

To study fully nonlinear properties of the gravito-electrostatic fluctuations of arbitrary amplitude, we apply the standard technique of the Sagdeev pseudo-potential approach, also known as the Sagdeev energy integral method (Sagdeev 1966; Mamun 1999; Shukla and Mamun 2002; Lakhina et al. 2008). The basic set of plasma governing equations (Eqs. (10)-(17)) is transformed into time-stationary form under the reference frame transformation, $\eta=\xi-\mu \tau$, where $\mu$ is the reference frame velocity (normalized by $c_{\mathrm{ss}}$ ). Then, we apply the appropriate boundary conditions $M_{i} \rightarrow$ $0, M_{e} \rightarrow 0, M_{d} \rightarrow 0, N_{i} \rightarrow 1, N_{e} \rightarrow 1, N_{d} \rightarrow 1, \Phi \rightarrow 0$, $\Psi \rightarrow 0, \partial \Phi / \partial \eta \rightarrow 0$ and $\partial \Psi / \partial \eta \rightarrow 0$ at $\eta \rightarrow \pm \infty$ for localized disturbances in our analytical integration scheme. Thus simplified, the coupled set of plasma structure equations gets transformed into a unique pair of new gravitoelectrostatically coupled energy integral equations set out as follows:

$\frac{1}{2}\left(\frac{\partial \Phi}{\partial \eta}\right)^{2}+V_{E}(\Phi)=0, \quad$ and

$\frac{1}{2}\left(\frac{\partial \Psi}{\partial \eta}\right)^{2}+V_{G}(\Psi)=0$,

where, $V_{E}(\Phi)$ and $V_{G}(\Psi)$ are known as the electrostatic and self-gravitational Sagdeev potentials, respectively. Their mathematical constructs are presented as follows:

$$
\begin{aligned}
V_{E}(\Phi)= & \frac{e^{2}}{\rho_{0} G m_{d}}\left[Z _ { i } n _ { i 0 } ( \frac { 1 } { 2 \mathrm { P } } ) \left\{\left(\beta \Phi-Z_{i} \beta\left(\frac{\Phi^{2}}{2}\right)+\mu \Phi\right.\right.\right. \\
& \left.-f_{i}^{\mathrm{eff}} \sqrt{\alpha} \eta_{i} \Phi\right)-\frac{1}{\left(2 Z_{i} \beta\right)}\left(-Q \sqrt{Q^{2}-(4 P R)}\right) \\
& \left.+\frac{1}{\left(Z_{i} \beta\right)}\left((2 P R) \ln \left(-Q+\sqrt{Q^{2}-(4 P R)}\right)\right)\right\}
\end{aligned}
$$




$$
\begin{aligned}
& -n_{e 0}\left(\frac{1}{2 A}\right)\left(2 \alpha \Phi-f_{e}^{\text {eff }} \sqrt{\alpha} \eta_{e} \Phi+\mu \Phi\right. \\
& \left.+\alpha \frac{\Phi^{2}}{2}\right)+n_{e 0}\left(\frac{1}{2 A}\right)\left\{\frac { 1 } { 2 \alpha } \left(B \sqrt{B^{2}-(4 A C)}\right.\right. \\
& \left.\left.+(4 A C) \ln \left(-B+\sqrt{B^{2}-(4 A C)}\right)\right)\right\} \\
& +n_{i 0}\left(\frac{1}{4 P \beta}\right) \\
& \times\left\{\left(\left(\beta+\mu^{2}\right) \sqrt{\left.\left(\beta+\mu^{2}\right)^{2}-\left(4 \mu^{2} P\right)\right)}\right\}\right. \\
& -n_{i 0}\left(\frac{1}{\beta}\right)\left\{\left(\mu ^ { 2 } \operatorname { l n } \left(\left(\beta+\mu^{2}\right)+\left(\left(\beta+\mu^{2}\right)^{2}\right.\right.\right.\right. \\
& \left.\left.\left.\left.-\left(4 \mu^{2} P\right)\right)^{1 / 2}\right)\right)\right\} \\
& +n_{e 0}\left(\frac{1}{4 A \alpha}\right)\left\{( 2 \alpha + \mu ) \left((2 \alpha+\mu)^{2}\right.\right. \\
& \left.\left.-\left(4 \mu^{2} A\right)\right)^{1 / 2}\right\}-n_{e 0}\left(\frac{1}{\alpha}\right)\left\{\mu^{2} \ln ((2 \alpha+\mu)\right. \\
& \left.\left.+\left(\left((2 \alpha+\mu)^{2}-\left(4 \mu^{2} A\right)\right)^{1 / 2}\right)\right)\right\} \\
& \left.+n_{d 0} \mu\left\{T^{2}-(\mu E-F) \ln \left(T^{2}+(\mu E-F)\right)\right\}\right],
\end{aligned}
$$

and

$$
\begin{aligned}
V_{G}(\Psi)= & \frac{m_{d} n_{d 0} \mu}{\rho_{0}}\left[\left\{F^{2}-(2 E G)\right\}^{1 / 2}\right. \\
& -[\mu E-F] \ln \left\{\left\{F^{2}-(2 E G)\right\}^{1 / 2}\right. \\
& +(\mu E-F)\}],
\end{aligned}
$$

where, the various involved coefficients are

$$
\begin{aligned}
A= & 4 \alpha, \quad B=-\left\{2 \alpha-f_{e}^{\mathrm{eff}}(\sqrt{\alpha}) \eta_{e}+\mu+\alpha \Phi\right\}, \\
C= & -\left\{\mu \eta_{e} \overline{N_{e}}\left(-f_{\mathrm{ed}}+f_{i}\right)-\beta_{\mathrm{eff} 0} n_{e 0} \eta_{e} \mu{\overline{N_{e}}}^{2}-\mu^{2}\right\}, \\
P= & 4 \beta, \quad Q=-\left\{\beta-Z_{i} \beta \Phi+\mu^{2}-f_{i}^{\mathrm{eff}}(\sqrt{\alpha}) \eta_{i}\right\}, \\
R= & \left\{\mu^{2}+f_{\mathrm{id}} \mu \eta_{i} \overline{N_{i}}-f_{i}\left(\frac{n_{e 0}}{n_{i 0}}\right) \eta_{e} \overline{N_{e}} \mu\right. \\
& \left.+\beta_{\mathrm{eff} 0}\left(\frac{n_{e 0}^{2}}{n_{i 0}}\right) \eta_{e} \mu{\overline{N_{e}}}^{2}\right\}, \\
E= & \left\{1+\left(\frac{T_{d}}{T_{e}}\right)\left(\frac{1}{\mu^{2}}\right)\right\} F=\left\{\mu-\left(\frac{T_{d}}{T_{e}}\right)\left(\frac{1}{\mu}\right)\right\}, \\
G= & \left\{f_{\mathrm{dn}} \sqrt{\frac{T_{d}}{T_{e}}} \eta_{d}+\frac{q_{d}}{e} \Phi+\Psi-F_{N}^{\mathrm{di}} \eta\right\}, \quad \text { and } \\
T= & \sqrt{\left\{F^{2}-(2 E G)\right\} .}
\end{aligned}
$$

Here, $\eta_{e}=\left(\eta_{e 1}-\eta_{0}\right), \eta_{i}=\left(\eta_{i 1}-\eta_{0}\right)$ and $\eta_{d}=\left(\eta_{d 1}-\eta_{0}\right)$ are the characteristic spatial variables (on the Jeans scale length) introduced for analytical simplification of the complex inhomogeneous dust cloud; and they are termed as average transit scale lengths of the electrons, ions and grains, respectively. Likewise, $\overline{N_{e}}$ and $\overline{N_{i}}$ are the spatially averaged number densities of the electrons and ions in normalized form, respectively.

In order to explore the basic characteristics of the fully nonlinear fluctuations, we carry out analytical tests to check the fulfillment conditions for the existence of possible structures like shocks, solitons, double layers and so forth (Sagdeev 1966; Mamun 1999; Shukla and Mamun 2002; Lakhina et al. 2008). Accordingly, we see that the electrostatic energy integral equation (Eq. (19)) satisfies the following extreme conditions meant for the existence of shock-like structures as follows:

$$
\begin{cases}V_{E}(\Phi)=0, \frac{\partial V_{E}(\Phi)}{\partial \Phi} \neq 0, & \text { at } \Phi=0, \\ \frac{\partial^{2} V_{E}(\Phi)}{\partial \Psi^{2}}<0, & \text { at } \Phi=0, \\ V_{E}(\Phi) \neq 0, & \text { at } \Phi=\Phi_{\max }, \text { and } \\ V_{E}(\Phi)<0 & \text { for } 0<|\Phi|<\left|\Phi_{\max }\right|\end{cases}
$$

On the other hand, the self-gravitational energy integral equation (Eq. (20)) satisfies the following conditions predicting the dynamical evolution of possible soliton-like patterns (Shukla and Mamun 2002; Lakhina et al. 2008) as follows:

$$
\begin{cases}V_{G}(\Psi)=0, \frac{\partial V_{G}(\Psi)}{\partial \Psi}=0, & \text { at } \Psi=0, \\ \frac{\partial^{2} V_{G}(\Psi)}{\partial \Psi^{2}}<0, & \text { at } \Psi=0, \\ V_{G}(\Psi)=0, & \text { at } \Psi=\Psi_{\max }, \text { and } \\ V_{G}(\Psi)<0 & \text { for } 0<|\Psi|<\Psi_{\max }\end{cases}
$$

It may be noted that the analytical conditions, as shown in Eqs. (23)-(24), for analytically characterizing the existence of eigenmodes, are obtained by using $\Phi=0, \Psi=0$, $T_{d} / T_{e}=0, \overline{N_{e}}=1, \overline{N_{i}}=1, f_{e}^{\text {eff }}=0, f_{\text {ed }}=0, f_{\text {id }}=0, f_{i}=$ $0, f_{i}^{\text {eff }}=0$ and $\beta_{\text {effo }}=0$. It is found that $V_{E}(\Phi)$ satisfies the shock-like structural evolution conditions (Eq. (23)) and $V_{G}(\Psi)$ satisfies the solitary evolution conditions (Eq. (24)). So, our analytical tests show possibility for the existence of electrostatic shock-like and self-gravitational soliton-like eigenmode structures.

\section{Results and discussions}

The main aim of the theoretical model presented here is to analyze the properties of fully nonlinear gravito-electrostatic eigenmodes in the presence of ion-drag forces included in the heavier dust grain dynamics in self-gravitating multifluid dusty plasma. It is analytically shown that the fluctua- 


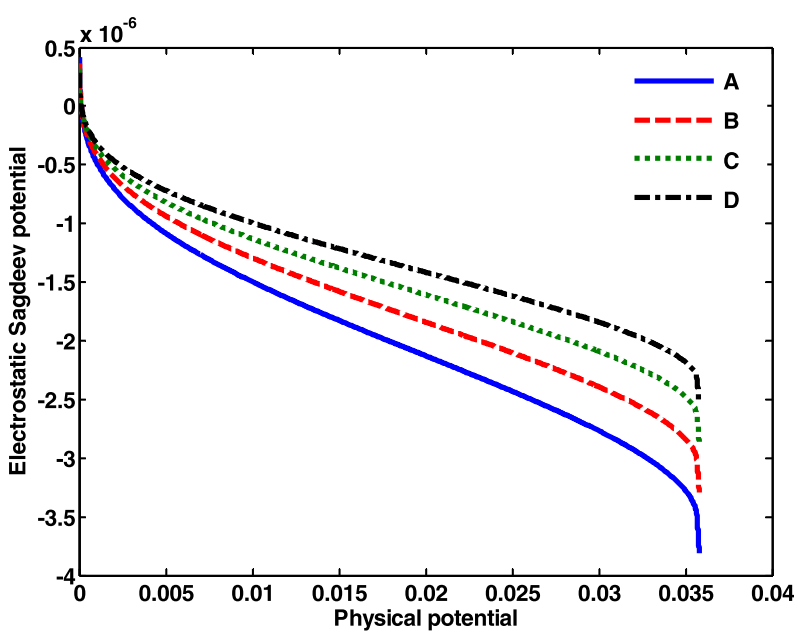

(a)

Fig. 1 Profile of the electrostatic (a) Sagdeev potential $V(\Phi)$ and (b) physical potential $\Phi$. Different lines correspond to Case (A): $m_{d}=4.0 \times 10^{-16} \mathrm{~kg}$ (blue line), Case (B): $m_{d}=4.3 \times 10^{-16} \mathrm{~kg}$

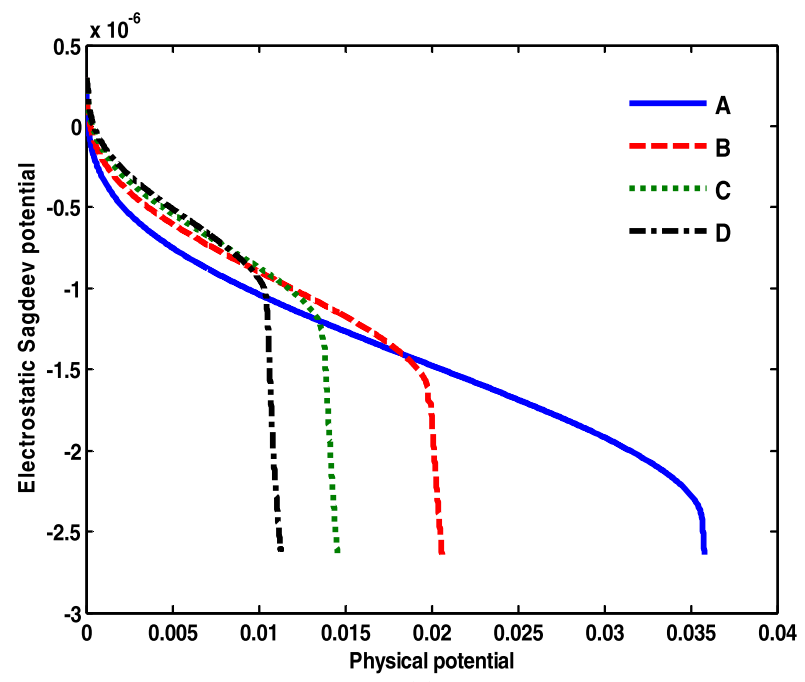

(a)

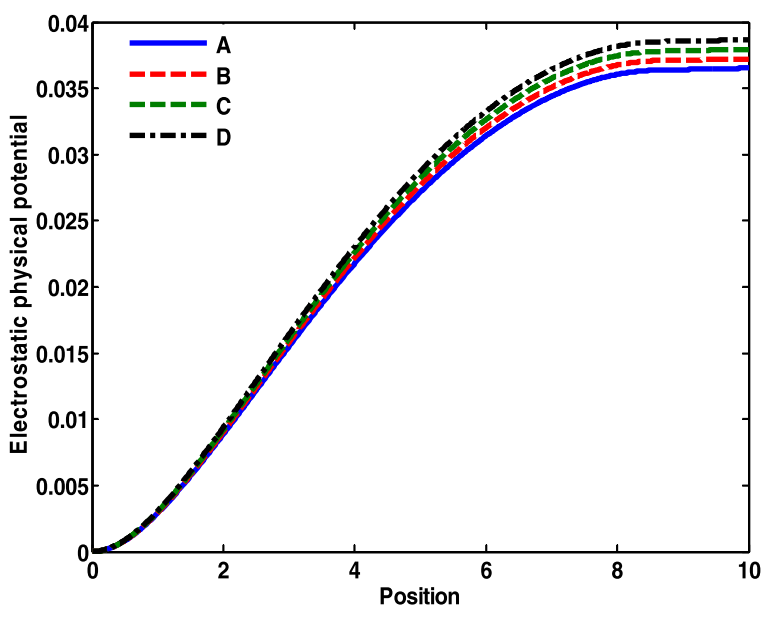

(b)

(red line), Case (C): $m_{d}=4.6 \times 10^{-16} \mathrm{~kg}$ (green line) and Case (D): $m_{d}=4.9 \times 10^{-16} \mathrm{~kg}$ (black line), respectively. Further inputs and details are discussed in the text.

Fig. 2 Same as Fig. 1, but in different conditions. Different lines correspond to Case (A): $Z_{d}=1.00 \times 10^{2}$ (blue line), Case (B): $Z_{d}=2.00 \times 10^{2}$ (red line), Case $(\mathrm{C}): Z_{d}=3.00 \times 10^{2}$ (green line) and Case $(\mathrm{D}): Z_{d}=4.00 \times 10^{2}$ (black line); respectively.

tions are governed by a new pair of gravito-electrostatically coupled energy integral equations (Eqs. (17)-(18)) by applying the Sagdeev pseudo-potential approach. In addition to approximate analytical features as already discussed above, the coupled system is analyzed numerically for exact solutions as initial value problems by the fourth-order RungeKutta method (RK-IV), a well established technique (Press et al. 2002). It is found by exact numerical integration that the collective fluctuations evolve as shock-like (electrostatic) and soliton-like (self-gravitational) patterns in judicious plasma parameter window (Goldston and Rutherford 1995) relevant for interstellar space as displayed graphically in Figs. 1, 2 and 3.

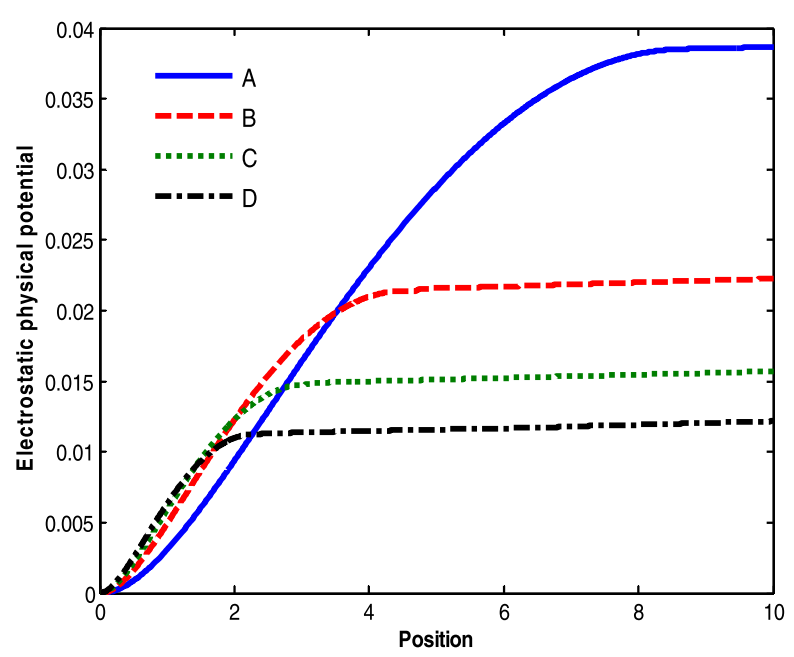

(b)
As shown in Fig. 1, the spatial profiles of electrostatic (a) Sagdeev potential and (b) physical potential depict the existence of unique class of shock-like structures supported in our DMC. Here, various lines correspond to Case (A): $m_{d}=$ $4.0 \times 10^{-16} \mathrm{~kg}$ (blue line), Case (B): $m_{d}=4.3 \times 10^{-16} \mathrm{~kg}$ (red line), Case (C): $m_{d}=4.6 \times 10^{-16} \mathrm{~kg}$ (green line) and Case (D): $m_{d}=4.9 \times 10^{-16} \mathrm{~kg}$ (black line); respectively. The other parameters kept fixed are $Z_{d}=100, n_{e o}=2.40 \times$ $10^{5} \mathrm{~m}^{-3}, n_{i o}=2.00 \times 10^{5} \mathrm{~m}^{-3}, n_{d o}=1.39 \times 10^{1} \mathrm{~m}^{-3}$, $m_{e}=9.1 \times 10^{-31} \mathrm{~kg}, m_{i}=1.67 \times 10^{-27} \mathrm{~kg}, \overline{N_{e}}=10^{3}$, $\overline{N_{i}}=10^{2}, f_{\text {id }}=2.00 \times 10^{-3}, Z_{i}=1, \eta_{e}=2.00 \times 10^{-2}$, $\eta_{i}=3.00 \times 10^{-2}, \eta_{d}=2.00 \times 10^{-2}, F_{N}^{\mathrm{di}}=1.00 \times 10^{3}$ (for subsonically streaming ions), $f_{\mathrm{dn}}=1.00 \times 10^{-2}, f_{\mathrm{ed}}=$ 


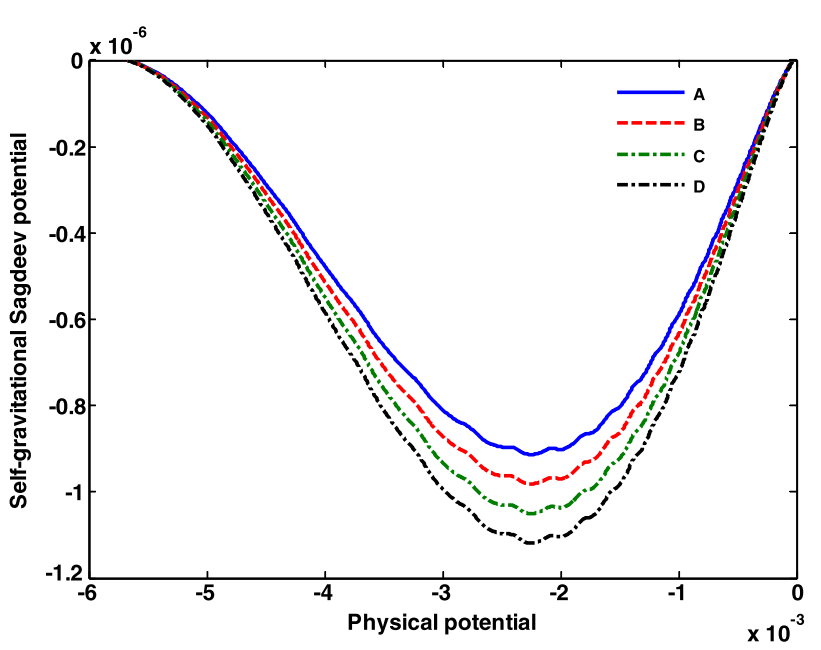

(a)

Fig. 3 Profile of the self-gravitational (a) Sagdeev potential $V(\Psi)$ and (b) physical potential $\Psi$. Different lines here correspond to Case (A): $m_{d}=4.00 \times 10^{-14} \mathrm{~kg}$ (blue line), Case (B): $m_{d}=4.30 \times 10^{-14} \mathrm{~kg}$

$1.00 \times 10^{2}, f_{\text {ion }}=1.00 \times 10^{2}, f_{i}=1.00 \times 10^{1}, f_{e}=$ $2.00 \times 10^{1}, \beta_{\text {eff } 0}=2.00 \times 10^{-3}$ and $\mu=1.02$. It is seen that the numerical patterns of $V_{E}(\Phi)$ (Fig. 1(a)) satisfy all the extreme analytical conditions given by Eq. (21). So, $\Phi$ must evolve as compressive shock-like eigenmodes, which is indeed so, as found numerically (Fig. 1(b)). It can be noticed that a finite value of $V_{E}(\Phi)$ exists at maximum wave amplitude, $\Phi=\Phi_{\max }$. Thus, in Fig. 1(b), we see, with proper numerical fitting, that the $\Phi$-fluctuations are convergent to $\Phi=\Phi_{\max }$ for the different increasing $m_{d}$-values. It is interesting to see that the $\Phi$-amplitude increases with increase in $m_{d}$, but the shock front-thickness remains the same (Fig. 1(b)). Thus, we can say that the increasing grain-mass has a destabilizing influential role on the electrostatic fluctuations. It may interestingly be pertinent to note that, when the $Z_{d}=100$ value is kept fixed, the $\Phi$-amplitude increase with the $m_{d}$-value is attributable to the large-scale ambipolar gravity-induced electrostatic polarization effects (Vranjes and Tanaka 2005). The basic physics behind it may easily be seen as in the following. An isolated isothermal dusty plasma gas in gravito-thermal equilibrium organizes itself in such a fashion that the heavier constituents (grains) preponderantly fill lower layers, leaving the lighter species (electrons and ions) distributed in upper layers. In the interstellar dusty conditions, the grain-to-ion scale heights may, with all the pre-defined usual notations, be compared as, $H_{d} / H_{i} \sim$ $\left(m_{d} / m_{i}\right)\left(T_{p} / T_{d}\right) \gg 1$. It indicates that a large-scale nonzero electrostatic potential is evolved in association with the gravity-induced stratification effects, which in turn, is responsible for the detected shock-features as in Fig. 1.

Likewise, Fig. 2 gives the same as Fig. 1, but with different conditions. Different lines correspond to Case (A):

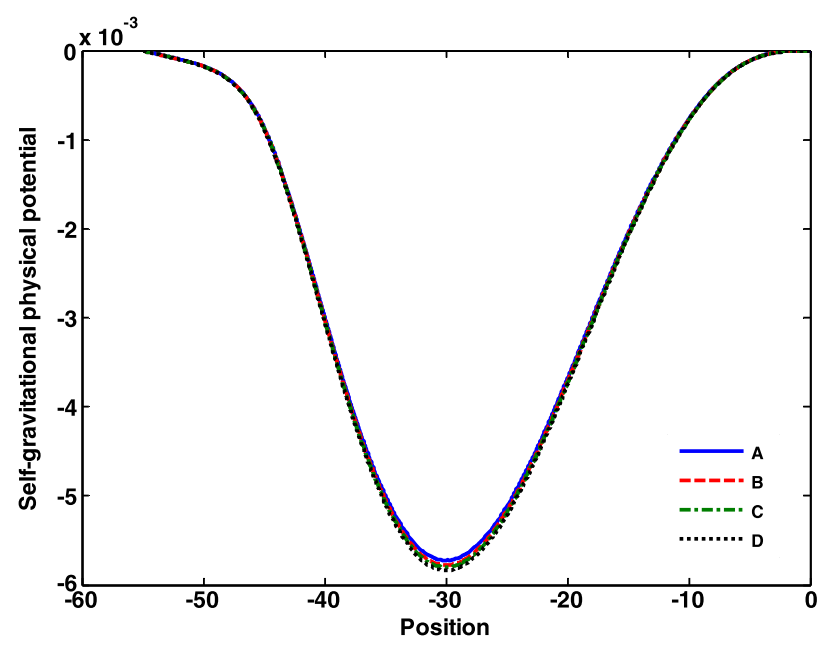

(b)

(red line), Case (C): $m_{d}=4.60 \times 10^{-14} \mathrm{~kg}$ (green line) and Case (D): $m_{d}=4.90 \times 10^{-14} \mathrm{~kg}$ (black line), respectively. Further inputs and details are given in the text.

$Z_{d}=1.00 \times 10^{2}$ (blue line), Case (B): $Z_{d}=2.00 \times 10^{2}($ red line), Case (C): $Z_{d}=3.00 \times 10^{2}$ (green line) and Case (D): $Z_{d}=4.00 \times 10^{2}$ (black line); respectively. In Fig. 2, we get similar structural patterns as shown in Fig. 1. Here, it is seen that the fluctuation amplitude of the compressive shock-like patterns decreases with increase in $Z_{d}$. This implies that the $Z_{d}$-increment introduces stabilizing effect for the electrostatic wave fluctuations. Now, we apply Eq. (18) in given plasma conditions to compare the relative strength of the ion-drag forces as, $\left(F_{N}^{\mathrm{di}}\right)_{1}:\left(F_{N}^{\mathrm{di}}\right)_{2}:\left(F_{N}^{\mathrm{di}}\right)_{3}:\left(F_{N}^{\mathrm{di}}\right)_{4}=1: 2:$ $3: 4$, for $Z_{d 1}=100, Z_{d 2}=200, Z_{d 3}=300$ and $Z_{d 4}=400$; respectively. It shows that the ion-drag force has a stabilizing influential role on the electrostatic wave propagation in the cloud, as well-reflected in Fig. 2 too. It parallelly indicates that if there is no ion-drag force effect, the cloud would electrostatically be more destabilized with far and far enhanced shock amplitudes. Thus, one can encounter that the ion-drag effects are important for re-organization of the plasma equilibrium, subjected to fully nonlinear perturbation against the equilibrium, where the observed compressive structures are supported.

In contrast, Fig. 3 graphically shows the spatial profiles of the self-gravitational (a) Sagdeev potential and (b) physical potential in some judicious plasma parameter space. It portrays the existence of soliton-like structures. Various lines correspond to Case $(\mathrm{A}): m_{d}=4.00 \times 10^{-14} \mathrm{~kg}$ (blue line), Case (B): $m_{d}=4.30 \times 10^{-14} \mathrm{~kg}$ (red line), Case (C): $m_{d}=4.60 \times 10^{-14} \mathrm{~kg}$ (green line) and Case (D): $m_{d}=$ $4.90 \times 10^{-14} \mathrm{~kg}$ (black line); respectively. The other parameters kept fixed are $Z_{d}=100, n_{d 0}=4.00 \times 10^{2} \mathrm{~m}^{-3}$, $F_{N}^{\mathrm{di}}=1.00 \times 10^{2}$ (for subsonic ion-flow), $\eta_{d}=2.00 \times 10^{-2}$, $f_{\mathrm{di}}=5.00 \times 10^{-3}, f_{\mathrm{dn}}=2.00 \times 10^{-2}$ and $\mu=10$. It is 
noticed that the numerical patterns (Fig. 2(a)) of $V_{G}(\Psi)$ also satisfy all the derived analytical conditions (Eq. (22)) required for the existence of solitary structures. Here, $\Psi$ evolves as rarefactive solitonary eigenmodes (Fig. 2(b)). It is seen that the amplitude of the eigenmodes increases with increase in the grain-mass, with the corresponding $V_{G}(\Psi)$ fluctuations merging to $\Psi=\Psi_{\max }$ (Fig. 2(b)). It is found that the $m_{d}$-increment plays destabilizing role for the selfgravitational wave fluctuations. This physically signifies that the self-gravitational potential has a direct correlation with the grain-mass. However, unlike the electrostatic fluctuations as already discussed, the $m_{d}$-independent ion-drag force effect does not play any significant role in influencing the dynamics of the self-gravitating cloud stability.

In order for quantitative description, the physical strength of the $\Phi$-fluctuations is calculated as $\phi \sim 5.35 \mathrm{~V}$ for average value of $\Phi \sim 3.5 \times 10^{-2}$ (Fig. 1(b)) with $T \sim 10^{4} \mathrm{~K}$. Similarly, the physical value of $\Psi$ is estimated as $\psi \sim$ $5.70 \times 10^{-3} \mathrm{~J} \mathrm{~kg}^{-1}$ for average value of $\Psi \sim 3.10 \times 10^{-8}$ (Fig. 3(b)) with $T \sim 10^{4} \mathrm{~K}$. It is clear from our analysis that the electrostatic potential fluctuations dominate over the self-gravitational counterpart within the framework of relevant interstellar plasma parameter space (Goldston and Rutherford 1995).

The analytical and the numerical findings in our investigation are in good correspondence with those obtained theoretically by others (Mamun 1999; Shukla and Mamun 2003; Lakhina et al. 2008). In addition, our results also go in good conformity with the laboratory-based experimental observations in different plasma situations existing in the literature (Luo et al. 1999; Reighard et al. 2006; Sheridan et al. 2008; Bandyopadhyay et al. 2008; Merlino et al. 2012).

Most importantly, our model results might be useful as theoretical support to explain the satellite observations on various nonlinear wave features existing in space and astrophysical environments (Gosling et al. 1968; Ergun et al. 1998a, 1998b; Franz et al. 1998; Lee et al. 2009; Pickett et al. 2003; Carley et al. 2013).

\section{Conclusions}

We theoretically study the properties of fully nonlinear wave dynamics sourced by gravito-electrostatic coupling, in collisional, unmagnetized and self-gravitational dust cloud with the help of the Sagdeev pseudo-potential method. The adopted model consists of the warm electrons, positive ions and negatively charged massive dust microspheres with partial ionization in presence of the ion-drag forces acting in anti-phase with the bipolar electrostatic forces arising due to ambipolar diffusive mechanism. All the realistic collisional effects, like between the electrons-ions, electronsgrains, ions-grains, etc., are simultaneously included. It is found that our model supports the existence of electrostatic compressive shocks and self-gravitational rarefactive solitons having wide-range stability significance in laboratory, space and astrophysical situations. To summarize, the main characteristic points on the fully nonlinear fluctuation dynamics investigated here are briefly concluded as follows:

1. A theoretical evolutionary model to see strongly nonlinear gravito-electrostatic fluctuations in astrophysical dusty plasma in presence of the ion-drag forces is developed.

2. A new pair of gravito-electrostatically coupled energy integral equations is systematically obtained by using the Sagdeev pseudo-potential approach.

3. Fully nonlinear fluctuations evolve as electrostatic compressive shocks and self-gravitational rarefactive solitons.

4. It is found that the strength of the electrostatic fluctuations is stronger than the self-gravitational counterpart $\left(\Phi / \Psi=0.6 \times 10^{1}\right)$ due to the considered self-gravitating grainy plasma environment.

5. The investigation on interplaying gravito-electrostatic coupling verifies the well-established fact that the gravity also induces finite non-zero electric field in a selfgravitating system via ambipolar gravity-induced electric polarization (Vranjes and Tanaka 2005) even in presence of streaming ions in the cloud.

6. Our results reveal that the electrostatic shock-like fluctuations evolve in the accelerating region $(\mu>1)$ of the DMC. Thus, it is likely to play an important role in understanding the mechanism of plasma-based particle energization in space and astrophysical environments (Pottelette et al. 1999).

7. The wave patterns investigated here match qualitatively with the results found experimentally in laboratories on dusty plasma systems (Luo et al. 1999; Reighard et al. 2006; Bandyopadhyay et al. 2008; Sheridan et al. 2008; Merlino et al. 2012). In addition, our results are in good agreement with different satellite observations like Viking, FAST, Freja, Polar, Cluster spacecraft, etc. (Gosling et al. 1968; Ergun et al. 1998a, 1998b; Franz et al. 1998; Lee et al. 2009; Pickett et al. 2003; Carley et al. 2013). The wave signatures are relevant in understanding collective plasma wave dynamics in various space and astrophysical regions, such as auroral zone, interplanetary space, polar magnetosphere, solar wind, etc.

8. Analysis shows that the ion-drag force has a stabilizing influential role on the electrostatic wave propagation in the cloud. It seems that if there is no ion-drag force effect the cloud would electrostatically be more destabilized with boost amplification in compressive shock amplitudes. Thus, we see that the ion-drag effects are important for re-organization of the plasma equilibrium, after suffering from fully nonlinear perturbation against the 
equilibrium, where the observed compressive structures are supported. In contrast, the $m_{d}$-independent ion-drag force effect does not play any significant role in influencing the self-gravitating cloud stability amid complex gravito-electrostatic interplay in the parameter windows explored in the study.

9. Lastly, our analysis seems to be useful to understand the particle-acceleration, material-transportation, clumpfilamentation instabilities significant in self-gravitational collapse of astrophysical clouds leading to the formation of stars and other galactic structures. Of course, we admit that further refinements are necessary to see the real evolutionary picture by inclusion of unavoidable factors like dust-charge fluctuations, magnetic field, rotational dynamics, non-Newtonian viscous-drag effect, etc.

Acknowledgements The worthful comments, specific remarks and precise suggestions by the anonymous referees to refine the scientific contents of the manuscript are gratefully acknowledged. The financial support from the Department of Science and Technology (DST) of New Delhi, Government of India, extended through the SERB Fast Track Project (Grant No. SR/FTP/PS-021/2011) is also thankfully recognized.

Open Access This article is distributed under the terms of the Creative Commons Attribution 4.0 International License (http://creative commons.org/licenses/by/4.0/), which permits unrestricted use, distribution, and reproduction in any medium, provided you give appropriate credit to the original author(s) and the source, provide a link to the Creative Commons license, and indicate if changes were made.

\section{References}

Bandyopadhyay, P., Prasad, G., Sen, A., Kaw, P.K.: Phys. Rev. Lett. 101, 065006 (2008)

Bergin, E.A., Hartmann, L.W., Raymond, J.C.: Astrophys. J. 612, 921 (2004)

Bittencourt, J.A.: Fundamental of Plasma Physics, 3rd edn. Springer, Berlin (2004). 200

Blandford, R.D., Ostriker, J.P.: Astrophys. J. 221, 29 (1978)

Burman, S., Chowdhury, A.R.: Chaos Solitons Fractals 14, 973 (2002)

Carley, E.P., et al.: Nat. Phys. 9, 811 (2013)

D’Angelo, N.: Phys. Plasmas 5(9), 3155 (1998)

Ergun, R.E., et al.: Geophys. Res. Lett. 25(12), 2041 (1998a)

Ergun, R.E., et al.: Geophys. Res. Lett. 25(12), 2025 (1998b)

Franz, J.R., Kintner, P.M., Pickett, J.S.: Geophys. Res. Lett. 25(8), 1277 (1998)
Goldston, R.J., Rutherford, P.H.: Introduction to Plasma Physics. Plasma Physics Laboratory, Princeton University Press, Princeton (1995)

Gosling, J.T., et al.: J. Geophys. Res. Space Phys. 73(1), 43 (1968)

Huba, J.D.: NRL Plasma Formulary. Naval Research Laboratory, Washington (2013)

Ivlev, A.V., Samsonov, D., Goree, J., Morfill, G.: Phys. Plasmas 6(3), 741 (1999)

Ivlev, A.V., Zhdanov, S.K., Khrapak, S.A., Morfill, G.E.: Plasma Phys. Control. Fusion 46, 267 (2004)

Ivlev, A.V., et al.: Astrophys. J. 723, 612 (2010)

Karmakar, P.K., Borah, B.: Phys. Scr. 86, 025503 (1) (2012)

Khare, A., Shukla, P.K.: Phys. Lett. A 189, 470 (1994)

Lakhina, G.S., Kakad, A.P., Singh, S.V., Verheest, F.: Phys. Plasmas 15, 062903 (2008)

Lee, E., Parks, G.K., Wilber, M., Lin, N.: Phys. Rev. Lett. 103, 031101 (2009)

Luo, Q.Z., D’Angelo, N., Merlino, R.L.: Phys. Plasmas 6(9), 3455 (1999)

Mace, R.L., Hellberg, M.A.: Planet. Space Sci. 41, 235 (1993)

Mamun, A.A.: Astrophys. Space Sci. 268, 443 (1999)

Mamun, A.A., Shukla, P.K.: Phys. Scr. 98, 107 (2002)

Merlino, R.L., Heinrich, J.R., Kim, S.-H., Meyer, J.K.: Plasma Phys. Control. Fusion 54, 124014 (2012)

Misra, A.P., Chowdhury, A.R.: Eur. Phys. J. D 32, 105 (2006)

Misra, A.P., Chowdhury, A.R., Chowdhury, K.R.: Phys. Lett. A 323, 110 (2004)

Misra, A.P., Chowdhury, K.R., Chowdhury, A.R.: Phys. Scr. 71, 207 (2005)

Pandey, B.P., Dwivedi, C.B.: J. Plasma Phys. 55, 395 (1996)

Pandey, B.P., Vranjes, J., Poedts, S., Shukla, P.K.: Phys. Scr. 65, 513 (2002)

Pandey, B.P., Holst, B.V.D., Vranjes, J., Poedts, S.: Pramāna 61, 109 (2003)

Paul, S.N., Roychowdhury, K., Burman, S., Roychowdhury, A.: Czechoslov. J. Phys. 56, 12 (2006)

Pickett, J.S., et al.: Nonlinear Proc. Geophys. 10, 3 (2003)

Pottelette, et al.: Geophys. Res. Lett. 26(16), 2629 (1999)

Press, et al.: Numerical Recipes in C, The Art of Scientific Computing, 2nd edn. (2002)

Rao, N.N., Shukla, P.K., Yu, M.Y.: Planet. Space Sci. 38(4), 543 (1990)

Reighard, A.B., et al.: Phys. Plasmas 13, 082901 (2006)

Sagdeev, R.Z.: Rev. Plasma Phys. 4, 23 (1966)

Sheridan, T.E., et al.: Phys. Plasmas 15, 073703 (2008)

Shukla, P.K., Mamun, A.A.: IEEE Trans. Plasma Sci. 29, 2 (2001)

Shukla, P.K., Mamun, A.A.: Introduction to Dusty Plasma Physics. Institute of Physics, Bristol (2002)

Shukla, P.K., Mamun, A.A.: New J. Phys. 5, 17.1 (2003)

Shukla, P.K., Silin, V.P.: Phys. Scr. 45, 508 (1992)

Vranjes, J., Tanaka, M.Y.: Phys. Scr. 71, 325 (2005)

Yaroshenko, V.V., Verheest, F., Morfill, G.E.: Astron. Astrophys. 461, 385 (2007) 